\title{
BMJ Open Barriers to accessing cancer services for adults with physical disabilities in England and Wales: an interview- based study
}

\author{
Dikaios Sakellariou, ${ }^{\oplus 1}$ Sally Anstey, ${ }^{1}$ Sarah Gaze, ${ }^{1}$ Eleri Girt, ${ }^{2}$ Daniel Kelly, \\ Barbara Moore, ${ }^{3}$ Sarah Polack, ${ }^{4}$ Roger Pratt, ${ }^{5}$ Gill Tyrer, ${ }^{6}$ Narelle Warren, ${ }^{7}$ \\ Wendy Wilkinson, ${ }^{2}$ Molly Courtenay ${ }^{1}$
}

To cite: Sakellariou D, Anstey S, Gaze S, et al. Barriers to accessing cancer services for adults with physical disabilities in England and Wales: an interviewbased study. BMJ Open 2019;9:e027555. doi:10.1136/ bmjopen-2018-027555

- Prepublication history and additional material for this paper are available online. To view these files, please visit the journal online (http://dx.doi. org/10.1136/bmjopen-2018027555).

Received 28 0ctober 2018 Revised 22 May 2019 Accepted 10 June 2019

Check for updates

(C) Author(s) (or their employer(s)) 2019. Re-use permitted under CC BY-NC. No commercial re-use. See rights and permissions. Published by BMJ.

For numbered affiliations see end of article.

Correspondence to Dr Dikaios Sakellariou; sakellarioud@cardiff.ac.uk

\section{ABSTRACT}

Objectives The aim of this study was to explore the barriers to accessing cancer services faced by adults with pre-existing physical disabilities.

Design Cross-sectional, exploratory qualitative study. Data were collected by semistructured interviews and analysed thematically.

Setting Participants were recruited through statutory and third sector organisations in England and Wales between October 2017 and October 2018.

Participants 18 people with a diagnosis of cancer and a pre-existing physical disability.

Results The findings illustrate that people with physical disabilities in England and Wales face a variety of barriers to accessing cancer services. The overall theme that emerged was that participants experienced a lack of attitudinal and institutional preparation both from healthcare professionals and healthcare facilities. This overall theme is illustrated through three subthemes: lack of acknowledgment of disability, unseeing disability and physical inaccessibility.

Conclusions As the population ages and increasing numbers of people live with cancer and disability, it is important to develop knowledge to respond to the needs of this population. The mere existence of services does not guarantee their usability. Services need to be relevant, flexible, and accessible and offered in a respectful manner It is important that healthcare professionals work towards inclusive healthcare provision, enabling the utilisation of services by all. Necessary steps to be taken include better communication between the various professionals and across the different teams involved in patients' care, raising awareness of how physical disability can affect or interact with cancer-related treatment and creating more accessible physical environments.

\section{INTRODUCTION}

Access to healthcare is a basic human right. It has several dimensions, including availability, affordability, relevance, physical accessibility, and approachability of services and their acceptability to service users. ${ }^{12}$ Guided by Levesque et $a l^{2}{ }^{2}$ in this article, we define access
Strengths and limitations of this study

- This is one of the first studies to explore barriers in access to cancer services for people with disabilities in the UK.

- We used an in-depth, iterative data collection design, whereby data analysis informed the interviews in order to explore the emerging themes in detail.

- Participants with a range of disabilities were included and suggestions are made about the development of cancer services that are inclusive.

- Most participants were over 50 years of age and, therefore, results may not be applicable to younger people.

to healthcare 'as the opportunity to reach and obtain appropriate healthcare services in situations of perceived need for care' ( $p$ 4), highlighting the interaction between embodied characteristics, social environment, and characteristics of the health system.

Evidence from across the world suggests that people with disabilities face barriers to accessing needed health services and experience poorer access to healthcare compared with the general population. ${ }^{3-8}$ People with disabilities often report that their needs are not understood, that they do not feel listened to, that they are perceived as patients of low priority due to their pre-existing condition(s), and that they face several barriers to accessing services. ${ }^{4910}$ The current research draws on these insights from disability studies to examine such barriers in a specific treatment context (ie, cancer services).

The available information suggests that people with disabilities may be less likely to be treated as effectively or as quickly compared with people without a disability. ${ }^{4}$ A systematic review on access to healthcare found that 'disabled people are restricted in accessing 
healthcare and report less satisfaction' (p 21). ${ }^{4}$ Difficulties in accessing healthcare can be caused by a range of barriers including lack of transport, inaccessible buildings, and intangible barriers, such as lack of disability awareness among healthcare professionals. ${ }^{49}$

There is also evidence from several countries, including the UK, the USA, and Australia that people with disabilities are less likely to use cancer screening services and report barriers in access to cancer services. ${ }^{11-17}$ Further to this, people with disabilities diagnosed with cancer report low satisfaction and use of services. ${ }^{13-15}$ Several studies have evidenced how access to some cancer screening services can be compromised for people with disabilities due to a range of physical, institutional, financial, and attitudinal barriers, ${ }^{13-17}$ leading to lower utilisation compared with people without a disability. ${ }^{17}{ }^{18}$ In an analysis of data from 71793 patients with cancer enrolled in the National Cancer Patient Experience Survey, Bone et al found that those with long-standing conditions in England, including people with physical conditions and disabilities, report poorer care compared with people with no disabilities. ${ }^{19}$

However, little is known about the reasons for these inequities and the specific barriers people with physical disabilities face to accessing cancer services. While research has been undertaken in the UK to explore the experiences of people with learning disabilities who develop cancer and organisational changes have been initiated in order to meet their needs, ${ }^{20} 21$ there has been limited research on the needs of people with physical disabilities who develop cancer. This lack of knowledge makes it difficult to improve services and ensure they are fully inclusive of all people, including people with disabilities. This is especially important considering that an estimated $69 \%$ of people diagnosed with cancer in the UK will have at least one other long-term condition. ${ }^{22}$ The majority of patients with cancer are over the age of 65 and it is likely that many will have multiple morbidities, which could often lead to disability. ${ }^{22}$

Our aim in this article is to explore the barriers experienced by adults with pre-existing physical disabilities who have had the additional experience of being diagnosed with and treated for a potentially curable cancer. We wanted to specifically focus on barriers in order to highlight the obstacles faced by people with disabilities as they seek to access healthcare, rather than discuss their experiences more generally. Guided by the Equality Act $2010,{ }^{23}$ we use the term people with pre-existing physical disabilities to refer to people who have a physical impairment that has a substantial and longterm (more than 12 months) negative effect on people's ability to carry out their usual daily life activities and which was present prior to the cancer diagnosis.

\section{METHODOLOGY}

\section{Study design and setting}

We used a cross-sectional, exploratory qualitative design to allow us to gain in-depth information on a previously under-researched topic. The research team consisted of qualitative health researchers, cancer clinicians, and two lay members. We used purposive sampling to recruit adults with any pre-existing physical disability who had experience of having been diagnosed and treated for any potentially curable cancer. Recognising the sensitivity of the issue and also wanting to encourage participants' reflections on their journey from beginning until a point of remission or cure, we sought to recruit people who had already experienced cancer and not those undergoing diagnosis or treatment at the point of recruitment. Information on the study, including an invitation to the study and contact information for the research team, was distributed via both cancer and disability organisations in England and Wales, including the Wales Cancer Network, Macmillan Voices, Tenovus Cancer Care, Healthwise Wales, Disability Wales, and the Spinal Injuries Association, and across hospital sites in Wales.

\section{Selection of participants}

Inclusion criteria

We used purposive sampling to recruit participants meeting the following criteria:

1. Have a pre-existing physical disability (self-reported).

2. Have a diagnosis of cancer, experience of surgery, radiotherapy and/or chemotherapy/hormone therapy and currently in remission or 6 months beyond treatment given with curative intent (self-reported), or be a significant other to a person with such a diagnosis.

3 . Be over 18 years of age.

4. Have the ability to communicate in English, with or without the use of assistive technology.

\section{Exclusion criterion}

1. People with non-melanoma skin cancer.

A member of the research team provided information about the study to prospective participants who made contact and sent them the participant information sheet, allowing them at least 2 days to read before deciding whether to participate.

\section{Data collection}

Data were collected via semistructured interviews, conducted in person or via the phone, between October 2017 and October 2018. We developed an interview guide (online supplementary file 1) and we used this loosely to structure the interviews. The guide was quite open to avoid guiding participants' answers. The opening question invited participants to talk about their physical disability and their cancer experience. Follow-up questions focused on topics such as physical accessibility of hospitals, transportation or their engagement in the decision-making process. Some sample questions included 'Have you faced problems or challenges accessing services?', and 'What kind of problems where these?'. Participants were invited to give in-depth information through the use of probing questions, such as 'When did 
this happen?', 'What happened next?', and 'How did you feel about that?'

Using an iterative process, data collection and analysis proceeded in parallel, with the interviews incorporating new information emerging from the analysis. The interviewer sought to gain in-depth information, by asking probing questions, by rephrasing the same question, and by asking for examples or for further clarification. We carried out 23 interviews, including follow-up interviews with five participants, seeking clarification on specific issues that arose in the analysis. Interviews lasted between 30 and $75 \mathrm{~min}$. Thirteen interviews were carried out over the phone and 10 face-to-face; we did not notice any differences in terms of duration and depth of information between these two different methods. There was no need for the use of augmentative and alternative communication, but two of the participants decided to be interviewed together with their partners. This is an often-used accommodation in disability research to enable participation. ${ }^{24}$ All interviews were audio recorded and transcribed verbatim.

\section{Data analysis}

Data were analysed thematically. The research team met every 4 months for the duration of the study, in order for the team to review and discuss the findings. Participants' accounts were first coded in large segments, which were grouped thematically following LeCompte and Schensul's recursive analysis strategy. ${ }^{25}$ Each newly collected data item was analysed and codes were developed; where new codes appeared, the previously coded data items were revisited and, where necessary, recoded until saturation was reached. The codes were reduced to themes following an inductive process involving SA, SG, and DS, whereby we discussed the essence of the codes and sought to develop themes that accurately captured the participants' experiences. Through this process, a thick description was generated which was used to elucidate the experiences of the participants. To increase the trustworthiness of the analysis, all transcripts were read and coded independently by two experienced qualitative researchers (SA and DS). Any differences in interpretation were resolved through discussion between these two researchers and the wider team. Furthermore, we sought validation of the emergent findings with some of the participants and they all agreed with the results of the analysis.

\section{Ethics}

The study was approved by the Research Ethics Committee at the School of Healthcare Sciences in Cardiff University and by the NHS Research Ethics Committee (reference: 17/WA/0153). Approvals were also granted by three health boards in Wales to advertise the study on hospital sites. Prospective participants were given full information through a participant information sheet, before deciding whether to participate, and signed a consent form. Confidentiality was maintained at all times. To further protect
Table 1 Demographic characteristics of the participants

\begin{tabular}{|c|c|c|c|}
\hline Pseudonym & Age & Sex & $\begin{array}{l}\text { Underlying health } \\
\text { condition leading to } \\
\text { impairment }\end{array}$ \\
\hline Sue & $56-65$ & $\mathrm{~F}$ & Multiple sclerosis \\
\hline Mary & $56-65$ & $\mathrm{~F}$ & Phocomelia \\
\hline Bob & $56-65$ & M & Heart disease, asthma \\
\hline Gavin & 56-65 & $\mathrm{M}$ & $\begin{array}{l}\text { Brain damage, heart } \\
\text { disease }\end{array}$ \\
\hline Kathryn & $56-65$ & $\mathrm{~F}$ & $\begin{array}{l}\text { Hemiplegia as a result of } \\
\text { brain haemorrhage }\end{array}$ \\
\hline Matthew & $46-55$ & M & Paraplegia \\
\hline Jane (partner) & $56-65$ & $\mathrm{~F}$ & $\begin{array}{l}\text { Tetraplegia as a result of } \\
\text { spinal cord injury }\end{array}$ \\
\hline Margot & $56-65$ & $\mathrm{~F}$ & Back injury \\
\hline Daniel & $66-75$ & M & Heart disease \\
\hline Lisa & $66-75$ & $\mathrm{~F}$ & Back injury \\
\hline Gregor & $66-75$ & M & $\begin{array}{l}\text { Back injury, chronic } \\
\text { obstructive pulmonary } \\
\text { disease }\end{array}$ \\
\hline Terry & $56-65$ & M & Neuropathy \\
\hline Stuart & $35-45$ & M & Osteoarthritis \\
\hline Emma & $56-65$ & $\mathrm{~F}$ & $\begin{array}{l}\text { Fibromyalgia, peripheral } \\
\text { neuropathy, back injury }\end{array}$ \\
\hline Lauren & $56-65$ & $\mathrm{~F}$ & $\begin{array}{l}\text { Cerebral palsy and } \\
\text { arthritis }\end{array}$ \\
\hline Sandra & $56-65$ & $\mathrm{~F}$ & $\begin{array}{l}\text { Hemiplegia as a result } \\
\text { of brain haemorrhage, } \\
\text { Meniere's disease }\end{array}$ \\
\hline $\begin{array}{l}\text { Robert } \\
\text { (partner) }\end{array}$ & $56-65$ & M & Vasculitis \\
\hline Judith & $46-55$ & $\mathrm{~F}$ & $\begin{array}{l}\text { Mobility impairment } \\
\text { as a result of brain } \\
\text { haemorrhage, arthritis }\end{array}$ \\
\hline
\end{tabular}

participants' identity, we do not report their exact age or the exact cancer site.

\section{Patient and public involvement}

Two lay team members were included in the research team. Both were people with physical disabilities who had cancer. They were recruited via the Wales Cancer Network and their role was to advise the broader team at all stages of the project and to assist with project development and management, analysis, and interpretation of the data.

\section{FINDINGS}

\section{Participants}

Table 1 describes the demographic details of each of the 18 participants.

Most of the participants (apart from Lisa, who received treatment 15 years prior to recruitment) received care in the National Health Service (NHS), or by private 
services subcontracted by the NHS, no more than 8 years preceding recruitment to the study. The majority of the experiences they shared related to primary care (mostly general practitioners) and to specialist inpatient cancer care, with some pertaining to secondary care (mostly accident and emergency).

The findings illustrate that people with physical disabilities in the UK face a variety of barriers to accessing cancer services. The overall theme that emerged from the data was that participants experienced a lack of adequate preparation both from healthcare professionals and healthcare facilities (ie, both at the level of the clinical encounter and also at the organisational level) to respond to their needs. This overall theme is illustrated through the following three, complementary and partially overlapping, subthemes: lack of acknowledgment of disability and the accommodations required, unseeing disability, and physical inaccessibility.

\section{Lack of acknowledgment}

Participants perceived there to be a lack of acknowledgment among health professionals about their disability-related needs. This was often accompanied by poor planning and unwillingness to properly consider the impact their actions could have on people with disabilities and an inability to be flexible and innovative with regard to the best way to support those with a disability. Lack of acknowledgment was a wide-ranging theme, encompassing issues pertaining to continuity of care, information sharing, and willingness to adapt.

Recounting his experiences of having an MRI scan in an out-of-hours private centre, Matthew, who was a wheelchair user, stated that:

They'd [healthcare professionals] never worked with anyone in a wheelchair ever. Had no idea how to get me into the MRI room, because of course my wheelchair is made of metal and they were panicking.

The wheelchair created what Matthew interpreted as a sense of panic, rather than eliciting a proactive and/or creative solution to get him into the MRI machine. Other participants recounted instances of strategic non-disclosure by healthcare professionals, associated with a lack of recognition that impairments may alter bodily states which need to be taken into account in planning for cancer treatment. For example, Emma started experiencing unexplained neurological symptoms soon after she started chemotherapy. Suspecting that the chemotherapy might have had an effect on her neuropathy, she raised her concerns with the consultant:

She [the consultant] said "Put it this way, if we told people all the things that may or may not happen after treatment with this particular drug, they wouldn't have it, and they would die'. So, she said 'We tell you what we know will definitely happen, but we don't tell you what might happen down the line', and I thought, I don't know how I feel about that, because
I like to make an informed choice, because it's me that has to live with the consequences of it or not live with them.

Participants felt that the various healthcare professionals involved in their care were only aware of specific issues related to their specialty and that these issues were not communicated to other members of the healthcare team involved in their care. In particular, participants reported a lack of communication between cancer specialists and specialists involved in the care related to their physical disability, leading to a lack of continuity of care. Participants indicated that they were often the ones who had to communicate their care needs to healthcare team members. Sue, for example, stated:

I think it was up to me to be sort of more proactive and say "oh what about this?" I think it would have been better if even, if whoever was dealing with me said, "should we look into this?"

Participants reported that sometimes their needs were recognised but ignored. A variety of reasons were given for this. Several participants talked about hospitals being understaffed and professionals overburdened. Seeking to explain poorer experiences of care, Mary, a power wheelchair user, talked about professionals viewing ' $a$ disabled person as second class', pointing to attitudinal barriers to receiving appropriate care.

Although most participants stressed the importance of professionals seeking their perspective and that of their families, this was not always done. As Jane noted:

But then nobody even looked at me and thought "do you know what, she's the expert on this man's body, because she's looked after it for 35 years. If she's saying something's not right, then something's not right." And nobody ever recognised that.

\section{Unseeing disability}

Several participants recalled experiences when healthcare professionals had failed to appreciate the waysuniquely shaped by their bodily state - in which they interacted with the world. The connecting thread across all the experiences included here was the perceived inability or lack of capacity of professionals to empathise with the embodied experience of living with a disability, which thus translated into an unwillingness to adapt their usual practice. Even when the physical presence of disability demanded attention, as in the case of people who used wheelchairs, participants reported having their impairment and any associated needs ignored. In effect, their disability was unseen, through not being considered during clinical decision-making in cancer care. It was unclear whether this was due to inadequate preparation of professionals to care for people with disabilities whose bodies did not conform to normative expectations, or perhaps, in the case of some participants, due to the hidden nature of some impairments and their effects. 
Consequently, this led to negative care experiences. For example, Jane described how her husband, Daniel, who had high-level tetraplegia, was repeatedly asked questions that he just could not answer:

The number of occasions when he was asked about symptoms that he might feel in his abdomen and he would say "but I have no sensation, how would I know?"

Healthcare professionals relied on self-reporting of symptoms, which, as in the example above, was often not possible. Daniel eventually developed sepsis but this was not diagnosed until it was too late, and he died. Although Jane knew Daniel was unwell, the lack of sensation meant that he was unable to detect and report the symptoms healthcare professionals would expect from someone who has sepsis.

The reported lack of consideration of disability had real effects on people. Lauren, who lived with mobility impairments as a result of cerebral palsy and arthritis, found professionals to be inadequately prepared to help her when she had to have an MRI scan:

I have to climb up onto the scan bed and I couldn't do that, and I had real trouble. I said, "there's a plastic chair over there, if you move that over here, I can put my foot on that and then I can lie on the scanner." Now, the difficult things for me, as I say, are sitting up and things like that, I couldn't do that, and they couldn't understand that (...) [they said] "oh you can't have the plastic chair," "well, can I just not use it to put my foot on?", "well, I suppose you could do, [but] you've got to lie down", "yes, I know I've got to lie down but I can't get upon the bed if you don't give me something to help me up."

There were also occasions when patients tried to flag up their specific impairment-related needs, only to be rebuked. Having had severe back pain due to spinal injury, Gavin was often not comfortable lying down and needed some adaptations. However, talking about a period spent in hospital following surgery to remove a tumour, he recounted the following incident:

Gavin: There was just one night nurse who was there for one night who insisted... [that] I wasn't in pain and it was all in the mind, and I will never forget her.

Interviewer: What happened there?

G: I was whingeing about the mattress because it was... I was in the high dependency unit, and I think it was an inflatable mattress. But, however I laid, I just couldn't get comfortable on this wretched mattress. But she was like, "it's a lovely mattress, you stop complaining."

Similarly, Mary was given permission to eat following surgery, but was left to eat alone, which was impossible due to her physical disability:
Being left to sort of try and eat something that I know I couldn't, and they were aware I couldn't do. So, when I eventually got this lovely meal that I'd been waiting for, it was stone cold so... it was cold but I was still going to eat it, you know, 'cos I was hungry.

\section{Physical accessibility}

Most of the participants experienced challenges related to the physical accessibility of hospital buildings and facilities. Even where certain provisions were in place, such as parking or staff to help people transfer in and out of the hospital, these were often inadequate. Matthew commented that:

I once went for an MRI in a building that was up a flight of steps, and to get into it I had to be taken into the subterranean basement where the garage was, down a huge steep ramp, over a giant grill that was where the water flowed down this huge ramp, and then through a car park to get into the building.

Parking was an issue that came up in most of the interviews and was universally experienced as stressful and problematic. Participants commented on the cost, which several could ill-afford and on the lack of adequate parking spaces. Bob stated that "parking is a bit of a nightmare', while Gregor said that:

I sometimes think, well, we could do with more disabled parking, but I don't know. Because with me having walking disabilities as well.

Getting to healthcare facilities was also challenging for some of the participants. Needing help with transfers or transportation, some participants wanted somebody to be there with them. As Kathryn said:

It is always a problem. And in fact, I've got a physio appointment at the local hospital later on this month, and my husband was working. (...) So, he's taking a day off now to take me.

Like Kathryn, several of the participants found they had to rely on family and friends to help them get to healthcare facilities. But even once in the healthcare facility, participants still faced problems.

Stuart: Waiting for porters to be moved around, because you literally can't go yourself, could be challenging, could be wearing, especially when it's painful to sit down anyway. (...) So, yeah, it was challenging getting up and down and being comfortably seated.

Often, participants found that while there was a system in place to help them navigate the hospital, this was often not sensitive to their specific needs, thus making them feel tired, or reinforcing their conviction that they need to be accompanied by a family member or friend, essentially limiting people's independence. 
Some participants also reported that once inside the hospital, it was sometimes uncomfortable to use screening equipment. Margot, for example, said that:

I do sometimes [have problems]. I will, because I can't stand straight, and I'm slightly crooked, and I generally lift a little bit; my hip goes towards the left and my shoulders go a bit towards the right. (...). People will then try to compensate as much as they can. But then, I understand as well that I have to be in the right position for them to do it.

The quote above foregrounds the expectation that people with disabilities are expected to conform to the requirements of screening equipment, often trying to literally fit into the available equipment. Inability to do so could be seen (by healthcare professionals and people with disabilities themselves) as a personal failure.

Some of the participants recounted incidents of service provision that was flexible and responsive to their needs. Emma, for example, highlighting how healthcare professionals helped her when she had an MRI, said:

When I have an MRI, if I'm in discomfort, they stop halfway through and get me out and help me to sit up and move about a minute, just to relax.

\section{DISCUSSION}

The findings of this study indicate that people with physical disabilities who go on to develop cancer, report several barriers to receiving acceptable levels of cancer care. Overall, participants perceived healthcare systems and staff to be inadequately prepared to address their needs. Participants felt that their physical disability, and the needs associated with it, were often unseen, despite its importance in their lives. Even when healthcare professionals acknowledged the existence of disability, participants found that professionals were not always adequately equipped or prepared to appropriately manage its effects on both their overall care and more specifically on their cancer-related care. Other obstacles reported by most of the participants were barriers going to and from the hospital and mobilising within healthcare facilities.

This is the first study to explore barriers in access to cancer services for people with disabilities in the UK from a disability studies perspective. As such, it complements epidemiological evidence on disparities in access to cancer screening for women with disabilities in the UK. ${ }^{171826}$ Recruitment was undertaken predominantly through cancer organisations and some disability organisations. This might have excluded people who identify as disabled but do not engage with cancer organisations. As this was an exploratory study about a little-explored issue, we included the perspectives and experiences of people with any physical disability (except frailty associated with increasing age) and all cancer diagnoses (except non-melanoma skin cancers), across all levels of service provision. This means we cannot link findings to specific impairments, cancer sites or services. Future research could focus on cancers that are more prevalent in people with disabilities, such as bladder cancer which is more common in people with spinal injury. ${ }^{27}$ Future research could also explore the perspectives of healthcare professionals on the barriers people with disabilities face and what could help improve their experiences. Finally, most of the participants in this study were aged over 55 years and some had several comorbidities, which may have impacted their experiences.

Participants recounted that disability often appeared to not be taken into account by healthcare professionals. Even in cases where, for example, participants mobilised using a wheelchair, they shared stories where healthcare professionals did not acknowledge their physical disability and additional needs related to it. While professionals typically have access to patients' medical notes, they may only focus on the issues related to cancer, or they may not know how to address disability-related issues. This concurs with findings from Kroll et al and Read et al, ${ }^{1028}$ who argue that people with disabilities do not always get what they need from their interactions with healthcare services. Participants in this study sometimes went through procedures that were not appropriate, or they were made to feel awkward for requiring adapted or additional services. Read et al argue that people with disabilities often need to alert healthcare professionals, or flag disability, so that their needs can be addressed. ${ }^{10}$ Some of the participants in our study aimed to do this, by mobilising strategies such as verbally alerting staff to their needs. However, requiring patients to self-identify as disabled is not always effective, as it might produce feelings of guilt in patients, or feelings that they are using extra resources, adding unnecessary burden to the system. ${ }^{10}$ Also, some people may not be in a position to make their needs known (eg, due to not feeling empowered to do so) and might suffer as a result.

Participants in this study reported physical inaccessibility as a challenge to accessing cancer services. This reflects the findings of previous studies that show physical inaccessibility to be a major factor negatively affecting utilisation of services. Iezzoni, ${ }^{11}$ in a study on access to diagnosis and treatment for breast cancer for women with mobility impairments, found that this population faced significant barriers related to access, including problems getting into clinicians' offices and using examination equipment, such as tables. Iezzoni found that the mere existence of accessible equipment does not guarantee its usability. ${ }^{11}$

Transportation was also a barrier for several of the participants, concurring with existing literature. Sakellariou and Rotarou, for example, ${ }^{6}$ found that people with disabilities in the UK had between 2 and 4 times higher odds (depending on impairment severity) of having unmet healthcare needs due to transportation barriers. Getting to and from hospital can be a complex undertaking, involving the coordination of family, friends, taxi drivers, hospital transport, and porters. The mere 
availability of transportation is not adequate; as Read et al argue ${ }^{10}$ hospital transportation is sometimes not wheelchair accessible or runs to a tight schedule, to accommodate as many people as possible. Participants in our study also commented that sometimes they had to allow an entire day for hospital appointments, as they had to wait for hours to be picked up before and after appointments.

Assessing the UK's compliance with the United Nations Convention on the Rights of Persons with Disabilities, the Committee on the Rights of Persons with Disabilities observed the existence of attitudinal, environmental, and institutional barriers preventing persons with disabilities from accessing healthcare. ${ }^{29}$ The reasons for these are far-reaching and intertwine: people with disabilities have lower rates of educational attainment, lower income, and lower employment rate, all of which are associated with lower use of healthcare services. ${ }^{30}{ }^{31}$ The structural disadvantages people face also have an intersectional dimension that cannot be ignored; we know, for example, that women with disabilities in the UK are more likely than any other group population group to face barriers to healthcare access. ${ }^{6}$

The findings of this study highlight several of the specific barriers faced by people with physical disabilities when they seek to access cancer services. Overall, the findings indicate that people with physical disabilities face significant barriers to accessing cancer services. While barriers relating to transportation and lack of acknowledgment of disability have been raised before, our findings also illustrate unseeing disability as a major barrier to accessing appropriate healthcare services.

Furthermore, the findings of this study illustrate a discrepancy between the experiences of patients and the recommendations of recent strategic documents, including 'Achieving world-class cancer outcomes: a Strategy for England 2015-2020' and the Cancer Delivery Plan for Wales. ${ }^{323}$ Both documents call for access to equitable care and promoting delivery of cancer care responsive to individual needs. In order to effectively address the needs of people with disabilities, it is necessary to move beyond an exclusively single-disease approach to cancer management. ${ }^{12}$ To do this, it is important to first understand the experiences and specific needs of this population, and then to develop resources to raise awareness and help services address these needs.

For healthcare services to be truly inclusive, they need to be relevant and patient centred, considering and not ignoring or minimising the disability and its effects on treatment. The WHO states that it is important to make 'existing healthcare systems more inclusive at all levels' so that people with disabilities can have equitable access to healthcare $(\mathrm{p} 7) .{ }^{3}$ It is important that healthcare professionals and health strategists work towards inclusive healthcare environments, acknowledging the existence of disability and enabling the utilisation of services by all. Necessary steps to be taken include enabling better communication between the different specialists involved in patients' care, raising awareness of how physical disability (including impairment-related medication) can affect or interact with cancer-related treatment, training healthcare professionals about strategies for monitoring symptoms for people unable to feel them, and striving for more accessible physical environments.

As Emma's story about what she found useful when undergoing an MRI scan illustrated, what is needed in order to achieve truly inclusive healthcare is a flexible way of service provision that is sensitive to the specific needs of each person. It is also important to include disability in the educational curricula of healthcare students, including nursing, medicine, and allied health professions. ${ }^{34}$ Rather than addressing this topic in one-off lectures that may otherwise not be connected with the rest of the curriculum, it is important for disability to be an integral part of health professionals' education. ${ }^{34}$ To achieve best outcomes, education on disability needs to include people with disabilities who can help students understand the lived experience of living with disability. ${ }^{35}$

\section{Author affiliations}

${ }^{1}$ School of Healthcare Sciences, Cardiff University, Cardiff, UK

${ }^{2}$ Wales Cancer Network, Cardiff, UK

${ }^{3}$ Health and Care Research Wales, Cardiff, UK

${ }^{4}$ International Centre for Evidence in Disability, London School of Hygiene and

Tropical Medicine, London, UK

${ }^{5}$ Lay Advisory Group Member, Cardiff, UK

${ }^{6}$ Lay Advisory Group Member, Llandudno, UK

${ }^{7}$ School of Social Sciences, Monash University, Melbourne, Victoria, Australia

Contributors DS and SA jointly designed the study, conceived the research question and aims and objectives and carried out the analysis. DS, SA and SG reviewed the literature and produced the analysis plan. SG and DS carried out data collection. All authors contributed to protocol development, data analysis and to drafting the manuscript and accepted the final version of the manuscript.

Funding This work was supported by Tenovus Cancer Care, Grant Number: TIG2017-05.

Competing interests None declared.

Patient consent for publication Not required.

Ethics approval The study was reviewed and approved by the Research Ethics Committee at the School of Healthcare Sciences in Cardiff University and by the NHS Research Ethics Committee (reference: 17/WA/0153).

Provenance and peer review Not commissioned; externally peer reviewed.

Data sharing statement Due the nature of the ethics approval received, we cannot make interview data publicly available.

Open access This is an open access article distributed in accordance with the Creative Commons Attribution Non Commercial (CC BY-NC 4.0) license, which permits others to distribute, remix, adapt, build upon this work non-commercially, and license their derivative works on different terms, provided the original work is properly cited, appropriate credit is given, any changes made indicated, and the use is non-commercial. See: http://creativecommons.org/licenses/by-nc/4.0/.

\section{REFERENCES}

1. Gulliford M, Figueroa-Munoz J, Morgan M, et al. What does 'access to health care' mean? J Health Serv Res Policy 2002;7:186-8.

2. Levesque JF, Harris MF, Russell G. Patient-centred access to health care: conceptualising access at the interface of health systems and populations. Int J Equity Health 2013;12:18.

3. World Health Organisation. Disabilities. No date. http://www.who.int/ topics/disabilities/en/ (Accessed 27 Sep 2018).

4. Gibson J, O'Connor R. Access to health care for disabled people: a systematic review. Social Care and Neurodisability 2010;1:21-31. 
5. Popplewell NT, Rechel BP, Abel GA. How do adults with physical disability experience primary care? A nationwide cross-sectional survey of access among patients in England. BMJ Open 2014;4:e004714.

6. Sakellariou D, Rotarou ES. Access to healthcare for men and women with disabilities in the UK: secondary analysis of cross-sectional data. BMJ Open 2017;7:e016614.

7. Rotarou ES, Sakellariou D. Inequalities in access to health care for people with disabilities in Chile: the limits of universal health coverage. Crit Public Health 2017;27:604-16.

8. Allerton L, Emerson E. British adults with chronic health conditions or impairments face significant barriers to accessing health services. Public Health 2012;126:920-7.

9. Drainoni ML, Lee-Hood E, Tobias C, et al. Cross-disability experiences of barriers to health-care access: consumer perspectives. J Disability Policy Studies 2006;17:101-15.

10. Read S, Williams V, Heslop P, et al. Being a disabled patient: negotiating the social practices of hospitals in England. Social Inclusion 2018;6:74-82.

11. lezzoni LI, Kilbridge K, Park ER. Physical access barriers to care for diagnosis and treatment of breast cancer among women with mobility impairments. Oncol Nurs Forum 2010;37:711-7.

12. Sarfati $D$, Koczwara B, Jackson $C$. The impact of comorbidity on cancer and its treatment. Cancer 2016;66:337-50.

13. Peters K, Cotton A. Barriers to breast cancer screening in Australia: experiences of women with physical disabilities. J Clin Nurs 2015;24(3-4):563-72.

14. Merten JW, Pomeranz JL, King JL, et al. Barriers to cancer screening for people with disabilities: a literature review. Disabil Health $J$ 2015;8:9-16.

15. Angus J, Seto L, Barry N, et al. Access to cancer screening for women with mobility disabilities. J Cancer Educ 2012;27:75-82.

16. Llewellyn G, Balandin S, Poulos A, et al. Disability and mammography screening: intangible barriers to participation. Disabil Rehabil 2011;33(19-20):1755-67.

17. Sakellariou D, Rotarou ES. Utilisation of mammography by women with mobility impairment in the UK: secondary analysis of crosssectional data. BMJ Open 2019;9:e024571.

18. Floud S, Barnes I, Verfürden M, et al. Disability and participation in breast and bowel cancer screening in England: a large prospective study. Br J Cancer 2017;117:1711-4.

19. Bone A, McGrath-Lone L, Day S, et al. Inequalities in the care experiences of patients with cancer: analysis of data from the National Cancer Patient Experience Survey 2011-2012. BMJ Open 2014;4:e004567.
20. Tuffrey-Wijne I, Bernal J, Hubert J, et al. People with learning disabilities who have cancer: an ethnographic study. Br J Gen Pract 2009;59:503-9.

21. Macmillan. Learning disabilities and cancer. Online resource. 2016 http://www.macmillan.org.uk/aboutus/healthandsocialcareprofe ssionals/newsandupdates/macvoice/spring2016/learningdisabilities andcancer.aspx (Accessed 26 Sep 2018).

22. Macmillan Cancer Support. The burden of cancer and other long term health conditions. 2015 www.macmillan.org.uk/documents/ press/cancerandotherlong-termconditions.pdf (Accessed 25 Sep 2018).

23. HM Government. Equality act. London: The Stationary Office, 2010.

24. Sakellariou D, Boniface G, Brown P. Using joint interviews in a narrative-based study on illness experiences. Qual Health Res 2013;23:1563-70.

25. LeCompte MD, Schensul JJ. Analyzing \& interpreting ethnographic data. Rowman Altamira, 1999.

26. Graham A, Savic G, Gardner B. Cervical and breast cancer screening in wheelchair dependent females. Spinal Cord 1998;36:340-4.

27. Gui-Zhong L, Li-Bo M. Bladder cancer in individuals with spinal cord injuries: a meta-analysis. Spinal Cord 2017;55:341-5.

28. Kroll T, Jones GC, Kehn M, et al. Barriers and strategies affecting the utilisation of primary preventive services for people with physical disabilities: a qualitative inquiry. Health Soc Care Community 2006;14:284-93.

29. Committee on the Rights of Persons with Disabilities. Concluding observations on the initial report of the United Kingdom of Great Britain and Northern Ireland. 2017 https://tbinternet.ohchr.org/ layouts/treatybodyexternal/SessionDetails1.aspx?SessionID=1158\& Lang=en (Accessed 15 Oct 2018).

30. Braithwaite J, Mont D. Disability and poverty: a survey of World Bank poverty assessments and implications. Alter 2009;3:219-32.

31. World Health Organisation. World Report on Disability. Geneva: World Health Organisation, 2011.

32. Independent Cancer Taskforce. Achieving World-Class Cancer Outcomes: a Strategy for England 2015-2020. 2016 http://www. cancerresearchuk.org/about-us/cancer-strategy-in-england (Accessed 26 Sep 2018).

33. Welsh Government. Together for health- cancer delivery plan; a delivery plan up to 2016 for NHS Wales and its partners. Cardiff: Welsh Government, 2012.

34. Shakespeare T, Kleine I. Educating health professionals about disability: a review of interventions. Health and Social Care Education 2013;2:20-37.

35. Shakespeare T, lezzoni LI, Groce NE. Disability and the training of health professionals. Lancet 2009;374:1815-6. 